\title{
Effects of flavor asymmetric media on pion and kaon properties
}

\author{
C. A. de Sousa and M. C. Ruivo \\ Departamento de Física da Universidade de Coimbra, P3000 Coimbra
}

We investigate the behaviour of pion and kaon properties in dense asymmetric and neutron matter in the context of a generalized three-flavor Nambu-Jona-Lasinio model, focussing mainly on Pauli blocking and Fermi sea effects. Two main features are discussed: the splitting between charged mesons and the appearance at a low density of low-lying modes which are excitations of the Fermi sea. The contribution of the low-lying modes to the satisfaction of PCAC relations becomes more and more important as the density increases.

\section{INTRODUCTION}

One of the important questions in intermediate and high energy physics is the understanding of strongly interacting matter as a function of temperature and density. It has been shown in the framework of some models that, at sufficiently high temperature and/or density, spontaneously broken symmetries are restored. The same restoration of symmetry is also expected to hold for the dynamically broken chiral symmetry in QCD. It is believed that this phenomenon could occur in heavy-ion collisions, the early universe and neutron stars. The properties of hadrons in the medium are expected to exhibit modifications which could be a signature of critical or pre-critical phenomena. Among hadrons, the low-mass mesons, specially those related to chiral symmetry breaking, play a significant role since they are closely related to vacuum properties. So, the problem of how the properties of the mesons change in nuclear matter in comparison to their free values has attracted a lot of attention recently. The Nambu-Jona-Lasinio model [1] is very successful in the description of the vacuum and mesons at zero and finite density or temperature $[2,3]$. However these studies were mostly confined to thermal effects and / or in-medium effects in flavor symmetric media.

Recently we have studied the behaviour of kaons in asymmetric quark matter simulating symmetric nuclear matter $\left(\rho_{u}=\rho_{d}, \rho_{s}=0\right)$. We have observed the splitting between $K^{ \pm}$and the occurrence of a low-lying $K^{-}$branch at a small baryonic density. This study has been extended to charged pions in neutron matter $\left(\rho_{d}=2 \rho_{u}, \rho=\rho_{u}\right)$ which exhibit a similar behaviour. Moreover we discuss the effects on the behaviour of kaon masses of introducing explicitly a certain amount of strange matter in the medium $\left(\rho_{u}=\rho_{d}, \rho_{s}=\right.$ $\frac{1}{4} \rho_{u}$ and $\rho=\frac{3}{4} \rho_{u}$ ) [5]. The possibility of pion and kaon condensation above some critical density, as suggested by Kaplan and Nelson [6], has also been analysed. 


\section{MODEL AND FORMALISM}

We adopt a three flavor version of the NJL model whose many-body Hamiltonian can be written in the form

$$
\begin{aligned}
H & =\sum_{i=1}^{N} \beta(i)[\boldsymbol{\gamma}(i) \cdot \boldsymbol{p}(i)+m] \\
& -\sum_{i \neq j} \delta\left(\boldsymbol{x}_{i}-\boldsymbol{x}_{j}\right) g \beta(i) \lambda_{a}(i) \beta(j) \lambda_{a}(j)\left[I-\gamma_{5}(i) \gamma_{5}(j)\right],
\end{aligned}
$$

where $\lambda_{a}(0 \leq a \leq 8)$ are the Gell-Mann flavor matrices, $\beta, \gamma_{5}, \gamma$ are Dirac matrices. The model parameters are: $m=\operatorname{diag}\left(m_{u}, m_{d}, m_{s}\right)$, the current quark mass matrix $\left(m_{d}=m_{u}\right)$, the coupling constant $g$ and the cutoff in three-momentum space $\Lambda$ which regularizes momentum space integrals.

The vacuum is described by a Slater determinant of negative energy states, $\left|\phi_{0}\right\rangle$. The homogeneous quark matter state is also described by a Slater determinant which now includes positive energy states up to the Fermi momentum $\lambda_{i}, i=u, d, s$. The density matrix for the homogeneous quark matter is given by $f=\operatorname{diag}\left(f_{u}, f_{d}, f_{s}\right)$ where

$f_{i}=\frac{1}{2}\left(1-\frac{\beta M_{i}+\boldsymbol{\alpha} \cdot \boldsymbol{p}}{\sqrt{p^{2}+M_{i}^{2}}}\right) \theta\left(\Lambda^{2}-p^{2}\right)+\frac{1}{2}\left(1+\frac{\beta M_{i}+\boldsymbol{\alpha} \cdot \boldsymbol{p}}{\sqrt{p^{2}+M_{i}^{2}}}\right) \theta\left(\lambda_{i}^{2}-p^{2}\right)$.

By minimizing the expectation value of the energy with respect to the constituent quark masses, $M_{i}$, a set of gap-like equations is obtained: $M_{i}=m_{i}-4 g<\bar{\Psi}_{i} \Psi_{i}>, i=u, d, s$, where $\left\langle\bar{\Psi}_{i} \Psi_{i}\right\rangle$ is the $i$ th quark condensate.

Mesons are collective excitations of the vacuum state described by $f_{i}(2)$. Using the framework of the Time Dependent Hartree-Fock formalism [2] we derive dispersion relations of the form

$1=-24 g \sum_{p}\left[\left(\frac{M_{u}\left(M_{i}-M_{u}\right)+\omega E_{u}}{\left[\left(\omega-E_{u}\right)^{2}-E_{i}^{2}\right] E_{u}}\right) \Theta_{u}+\left(\frac{M_{i}\left(M_{u}-M_{i}\right)-\omega E_{i}}{\left[\left(\omega+E_{i}\right)^{2}-E_{u}^{2}\right] E_{i}}\right) \Theta_{i}^{\prime}\right]$,

with $\Theta_{u}=\theta\left(\Lambda^{2}-p^{2}\right)-\theta\left(\lambda_{u}^{2}-p^{2}\right)$ and $\Theta_{i}^{\prime}=\theta\left(\Lambda^{2}-p^{2}\right)-\theta\left(\lambda_{i}^{2}-p^{2}\right)(i=s, d)$.

Equation (3) with $i=s$ and $\omega=m_{K^{+}}$allows for the change of $K^{+}$mass with density, while the corresponding expression for $K^{-}$is obtained by replacing $\omega \rightarrow-m_{K^{-}}$. Due to the quark content $\bar{d} u(\bar{u} d)$ for $\pi^{+}\left(\pi^{-}\right)$in comparison with $\bar{s} u(\bar{u} s)$ for $K^{+}\left(K^{-}\right)$ expression (3) also allows for pions when $i=d$.

\section{KAONS AND PIONS IN DENSE MATTER}

There are two types of solutions of the dispersion relations (see Eq. (3)): discrete bound state and continuum solutions [5]. Only the bound state solutions are explicitly studied in this paper. The allowed discrete bound states are of two kinds: particle-antiparticle modes driven by the Dirac sea, already present in vacuum and associated with the dynamical chiral symmetry breaking; and particle-hole excitations of the Fermi sea, that only appear in the medium and are associated with the breaking of the flavor symmetry. 


\subsection{Kaons in strange matter}

The behaviour of kaon masses in matter with strange quarks $\left(\rho_{u}=\rho_{d}, \rho_{s}=\frac{1}{4} \rho_{u}\right)$ is shown in Fig. 1. The $K^{+}$mode is a collective $u \bar{s}$ excitation of the Dirac sea and no Fermi sea effects of particle-hole excitations contribute for this excitation. The increasing of $K^{+}$ mass is mainly due to repulsive effects of Pauli blocking. The effects of Fermi sea are seen above the threshold density $\rho_{t h}=0.4 \rho_{0}$, showing that the continuum band of particlehole excitations of the Fermi sea induces attractive (repulsive) correlations below (above) its boundary. Consequently, above this threshold density the $K^{-}$Dirac sea excitation is an increasing function of the density, while a low-lying $K^{-}$mode, an excitation of the Fermi sea, appears and is a decreasing function of $\rho$. One observes that the kaon-like $s \bar{u}$ particle-hole excitations play here a role similar to that of hyperon-particle- nucleon-hole excitations found in [7]. However one should not forget that in our case the Fermi sea is a gas of quarks and so our considerations concerning a gas of baryons can only be made through a simple minded extrapolation. This behaviour is qualitatively similar to those found in symmetric nuclear matter [4]. The presence of strange matter does not change qualitatively the results provided $\rho_{s}<\rho_{u}$. As a matter a fact only when the medium is flavor symmetric $\left(\rho_{u}=\rho_{d}=\rho_{s}\right)$ the low energy mode and the $K^{ \pm}$splitting will disappear.

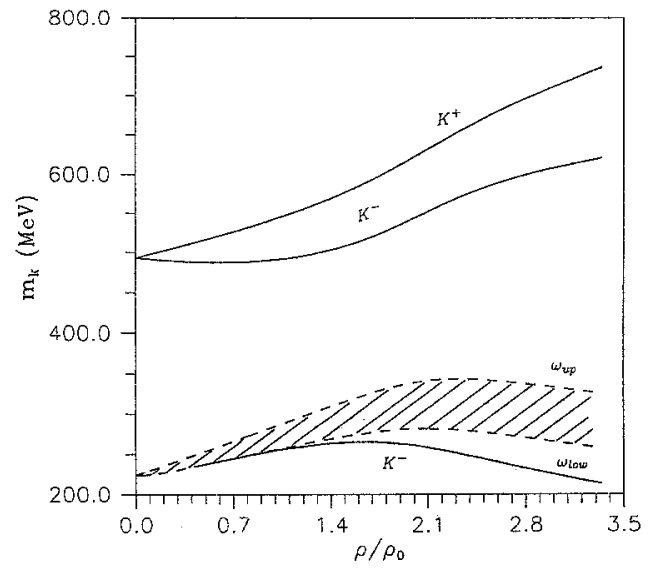

Figure 1. Kaon masses in strange matter as a function of baryonic density. The hatched area indicates the particle-hole continuum of the Fermi sea.

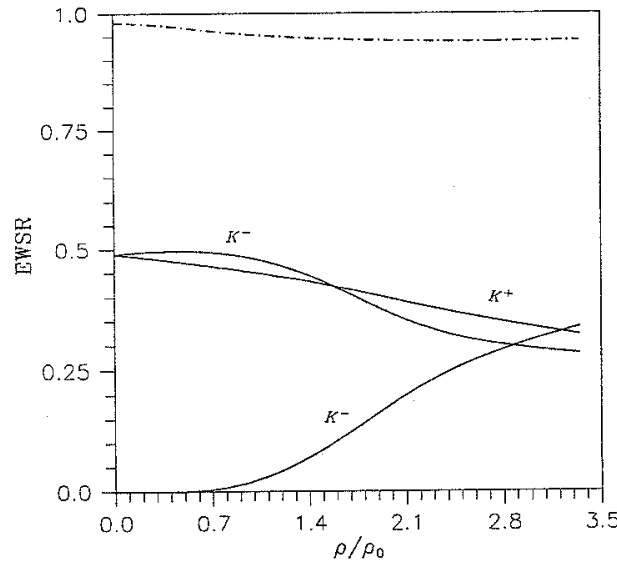

Figure 2. Percentages of the EWSR exhausted by the bound state solutions in strange matter. The sum of these percentages is represented by the upper line.

The ratios $f_{K, \alpha}^{2} m_{K, \alpha}^{2} /\left[-\left(m_{s}+m_{u}\right)\left(<\bar{\Psi}_{u} \Psi_{u}>+<\bar{\Psi}_{s} \Psi_{s}>\right)\right]$ for the kaon modes, which give us information about the degree of satisfaction of the Energy Weighted Sum Rule (or PCAC) and of the strength associated to each mode, are presented in Fig. 2. The sum of the percentages of the Energy Weighted Sum Rule (EWSR), represented by the dashed-dotted line, is situated around $100 \%$, showing that the PCAC relation is very well satisfied even when in-medium effects are included. The presence of the low-lying 
$K^{-}$mode is essential to the satisfaction of the sum rule and becomes more important as the density increases.

\subsection{Pions in neutron matter}

The neutron matter environment $\left(\rho_{d}=2 \rho_{u}\right)$, induces a reduction of the phase space with consequences in the behaviour of pion masses. One observes that the main features refered in the last subsection are found in this case. In fact, in spite of the attraction due to a decreasing of $d$ quark mass with density, the competition with repulsive Pauli blocking effects acts increasing the $\pi^{-}$mass. The existence of two discrete bound state solutions occurs now for modes with $\pi^{+}$quantum numbers. The low-lying $\pi^{+}$mode is a isospin excitation associated with the breaking of the $S U(2)$ symmetry, which appears at $\rho_{n}=0.3 \rho_{0}$ with the value $18.7 \mathrm{MeV}$. This Fermi sea $\pi_{s}^{+}$branch is situated below the Fermi sea continuum and decreases with neutron density. Also in this case, we notice that the EWSR is satisfied at the $98 \%$ level if the three $\pi^{-}, \pi^{+}$and $\pi_{s}^{+}$modes are considered.

\section{CONCLUSIONS}

This work is mainly concerned with effects of flavor asymmetry on kaons and pions. It is observed that both the $K^{+}$and $\pi^{-}$masses in flavor asymmetric matter increase with density mainly due to the repulsive effects of Pauli principle. Both $K^{-}$and $\pi^{+}$masses in the same scenarios depend on the combined Dirac and Fermi sea effects. These last effects are repulsive above the threshold density and also induce low-lying modes which are a manifestation of the complexity of the many-body system. We remark that, while the $K^{ \pm}\left(\pi^{ \pm}\right)$modes are excitations of the Dirac sea, the low-lying $K^{-}\left(\pi_{s}^{+}\right)$mode, a non-trivial dynamical consequence of the flavor symmetry breaking of the medium, is a collective particle-hole excitation of the Fermi sea. We point out that the repulsive effects of the Fermi sea allow for important non-perturbative features which do not favour boson condensation.

Work supported in part by GTAE and by projects CERN/S/FIS/1034/95, PRAXIS/2/ 2.1/FIS/451/94, ESO PESO/S/PRO/1057/95 and PRAXIS/PCEX/P/FIS/13/96.

\section{REFERENCES}

1. Y. Nambu and G. Jona-Lasinio, Phys. Rev., 122 (1961) 345; 124 (1961) 246.

2. J. da Providência, M. C. Ruivo and C. A. de Sousa, Phys. Rev. D 36 (1987) 1882; C. A. de Sousa, Z. Phys. C 43 (1989) 503; M. C. Ruivo, C. A. de Sousa, B. Hiller and A. H. Blin, Nucl. Phys. A 575 (1994) 460.

3. S. P. Klevansky, Rev. Mod. Phys. 64 (1992) 649.

4. M. C. Ruivo and C. A. de Sousa, Phys. Lett. B 385 (1996) 39.

5. C. A. de Sousa and M. C. Ruivo, Europhys. Lett. 37 (1997) 609; C. A. de Sousa and M. C. Ruivo, Coimbra preprint (1997)

6. D. B. Kaplan and A. E. Nelson, Phys. Lett. B 175 (1986) 57; B 192 (1987) 193.

7. H. Yabu, S. Nakamura, F. Myhrer and K. Kudodera, Phys. Lett. B 315 (1993) 17. 